\title{
Electronic and Biologic Simulations of Fetal Heart Rate Changes
}

\section{Kazuo Maeda*}

Department of Obstetrics and Gynecology (Emeritus), Tottori University Medical School, Yonago, Japan

The simulation is an analyzing technique of medical phenomena with electronic or biologic models to acquire similar results of the phenomena. Electronic system utilized in the simulation presents the developing mechanism of changes. The simulations were studied here on the developmental process of fetal heart rate (FHR) changes.

\section{Development of FHR Variability (Long Term Variability, LTV)}

Short term variability (STV) is the beat-to-beat variation in the instantaneous heart rate meter, and long term variability (LTV) is the FHR baseline fluctuation of common ultrasonic Doppler FHR monitor, i.e. the development of FHR changes in common fetal monitor is the subject of this report.

The moderate LTV develops in the benign physiologic sinusoidal FHR, while the pathologic sinusoidal one results fetal or neonatal death. The physiologic sinusoidal FHR was differentiated from the pathologic one by the associated periodic fetal respiratory movement recorded by the acto-cardiogram (ACG) (Figure 1). In other words, the FHR develops physiologic sinusoidal change provoked by the periodic fetal respiratory movements, i.e. LTV is the fetal reaction to fetal movement. Small baseline variability develops by the minor fetal movements [1]. Since the correlation coefficient of FHR and fetal movements was maximal when the movement delayed for $7 \mathrm{sec}$ [2], the FHR change appeared $7 \mathrm{sec}$ after the fetal movement.

\section{FHR Acceleration}

The triangular FHR acceleration synchronized to the group of large fetal movements (fetal movement burst) (Figure 2) [1], i.e. the movement burst stimulate fetal brain to provoke the acceleration, which develops in mid-brain in the studies on the anencephalic fetus [3]. Why the shape of acceleration is triangular? As the electric square wave changed to triangular wave after passing through an integral circuit, grouped $10 \mathrm{~Hz}$ waves of $30 \mathrm{sec}$ duration, which was similar to fetal movement bursts, was passed through an integral circuit, of which time constant (delay time) was $7 \mathrm{sec}$, then the output of integral circuit was triangular (Figure 3), i.e. the mid-brain will responded fetal movement bursts by an integral system with 7 sec delay. Adult continuous leg motion for $1 \mathrm{~min}$ developed triangular heart rate changes (Figure 4), which will show the conversion of movement bursts to triangular heart rate in the mid-brain.

\section{The Brain Damage in the Loss of FHR Variability}

Since the fetal movement baseline of ACG was not flat but filled

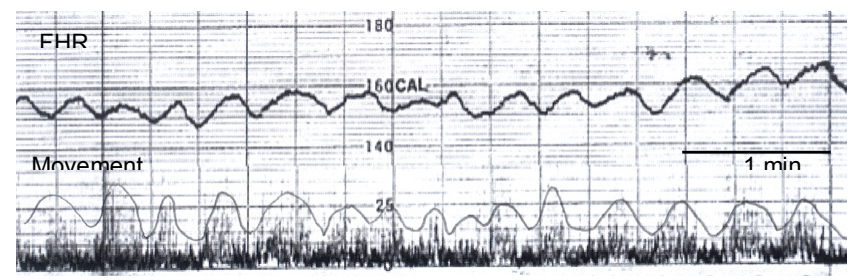

Figure 1: Fetal periodic respiratory movements (lower line) provoked moderate long term HR variability as a physiologic sinusoidal FHR pattern (upper line). with minor movement signals in augmented ACG, FHR variability was provoked by the reaction of mid-brain to minor movements [1]. FHR acceleration and LTV develop in the same center of the mid-
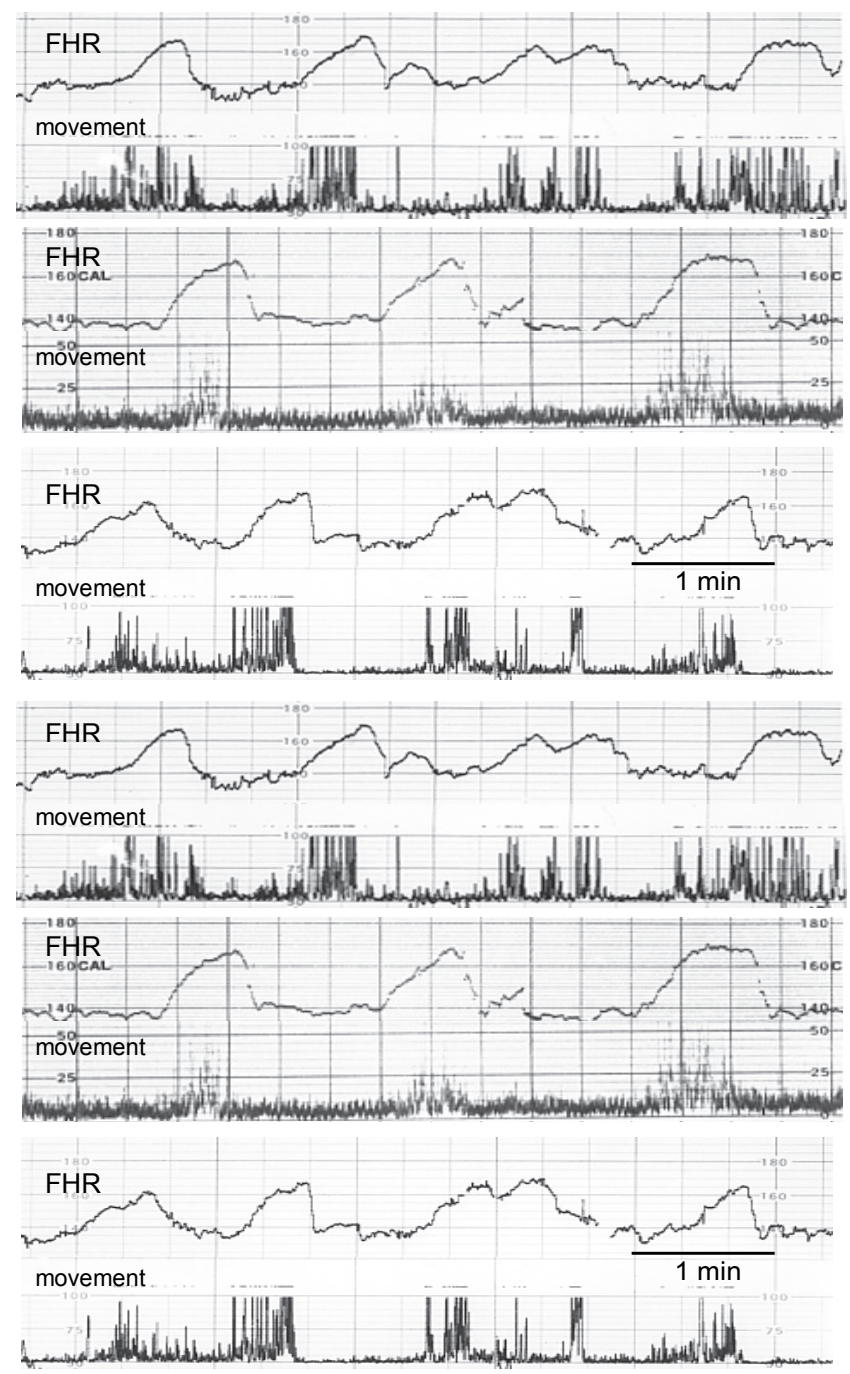

Figure 2: FHR acceleration is triangular, and provoked by the fetal movement burst.

*Corresponding author: Kazuo Maeda, Department of Obstetrics and Gynecology (Emeritus), Tottori University Medical School, Yonago, Japan, 3-125, Nadamachi, Yonago, Tottoriken, 6830835, Japan, Tel: 81-859-22-6856; Fax: 81-859-22-6856; E-mail:maeda@mocha.ocn.ne.jp

Received November 04, 2013; Accepted November 06, 2013; Published November 10, 2013

Citation: Maeda K (2014) Electronic and Biologic Simulations of Fetal Heart Rate Changes. J Health Med Informat. 5: e117. doi:10.4172/2157-7420.1000e117

Copyright: (c) 2014 Maeda K. This is an open-access article distributed under the terms of the Creative Commons Attribution License, which permits unrestricted use, distribution, and reproduction in any medium, provided the original author and source are credited. 


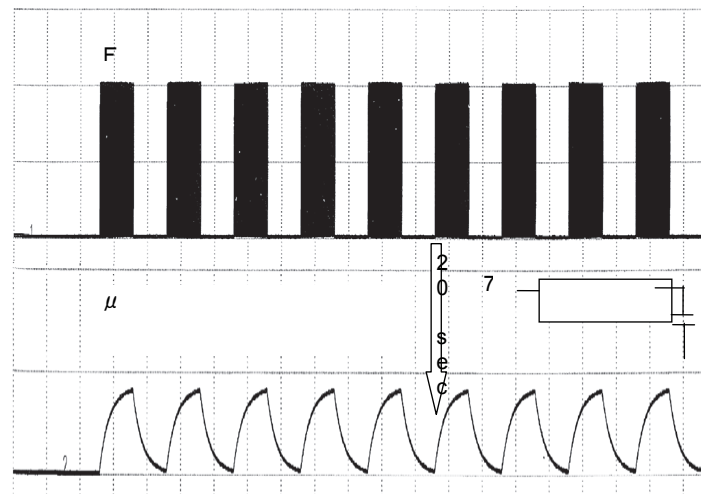

Figure 3: The electronic simulation to form triangular waves from burst-like 10 $\mathrm{Hz}$ wave groups passing through $7 \mathrm{sec}$ delay time constant integral circuit.

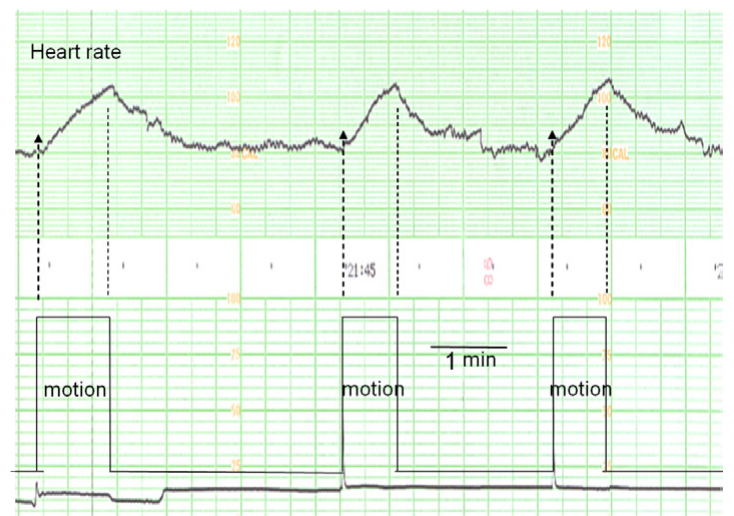

Figure 4: Biological simulation to develop triangular heart rate by one min long lasting leg motion.
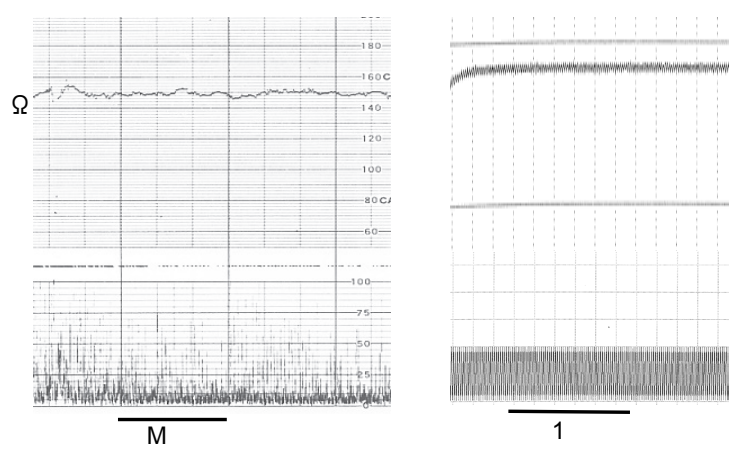

Figure 5: FHR develop no acceleration (A) by the long lasting fetal hiccupping motion (B).

brain, developing large acceleration by large movement burst, and small baseline variability from minor fetal movement. Therefore, severe loss of LTV with the loss of acceleration will be the sign of fetal brain damage. The nonreactive FHR has no acceleration against movement bursts, but preserved LTV[4], which means the suppression of fetal brain but not total loss of brain function, while some days later, severe NRFS appears showing bradycardia or severe decelerations, and the loss of LTV similar to anencephalic FHR, which means the brain damage including neuronal cell necrosis followed by possible cerebral

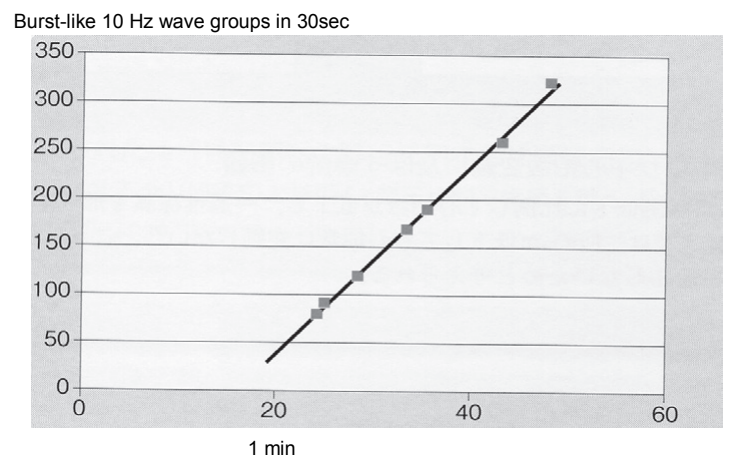

Figure 6: Rabbit heart rate is closely correlated $\mathrm{PaO}_{2}\left(R^{2}=1\right)$ [5]. Such close correlation as the rabbit is not measured and vague in human fetus, i.e. the correlation is a hypothesis, but that is the basic logic of hypoxia index.

palsy (CP), i.e. outcome of NRFS in non-reactive FHR outcome was ominous, resulting neonatal death despite the cesarean section (C-section)indicated by the NRFS [4]. Effective C-section to prevent CP will be performed before development of the loss of LTV. Since the loss of FHR variability in non-hypoxic fetal disorders also will be the sign of fetal brain damage, C-section should be made before the loss of variability too.

\section{Fetal Hiccupping Movements}

The FHR variability was preserved but there was no acceleration in the continuous fetal hiccupping movements, which appeared with 1-3 sec intervals (Figures 5A and 5B). In electronic simulation, the phenomena was simulated by the passing of continuous $1 \mathrm{~Hz}$ signals through the integral circuit with $7 \mathrm{sec}$ time constant ( $7 \mathrm{sec}$ delay system) resulting no acceleration with the elevation of baseline (Figures $5 \mathrm{C}$ and $5 \mathrm{D})$. The simulation confirmed the presence of an integral system in the mid-brain with the delay for $7 \mathrm{sec}$.

The loss of acceleration in continuous fetal hiccupping was confirmed by an electronic simulation, i.e. continuous one $\mathrm{Hz}$ signals (D) develop no acceleration (C) passing through an integral circuit with $7 \mathrm{sec}$ delay time constant. The presence of integral system with $7 \mathrm{sec}$ delay in the mid-brain is confirmed by the electronic simulation technique.

\section{Fetal Bradycardia in Hypoxia}

Fetal brain is suppressed, paralyzed and damaged by the progress of hypoxia, but the response of medulla oblongata was opposed, i.e. the vagal center located in medulla oblongata was excited by the deficiency of oxygen, hypoxia, and develop the decrease of heart rate, bradycardia. The two opposite reactions of large brain and medulla oblongata were mixed and showed complicated FHR patterns in hypoxia.

FHR showed gradual decrease along with the rabbit's $\mathrm{PaO}_{2}$ reduction in the nitrogen gas inhalation hypoxia (Figure 6). The bradycardia was recognized when rabbit's $\mathrm{PaO}_{2}$ was lower than $50 \mathrm{mmHg}$ [5]. Since fetal umbilical arterial $\mathrm{PaO}_{2}$ was lower than $50 \mathrm{mmHg}$, the FHR will be linearly decreased along with the low fetal $\mathrm{PaO}_{2}$ as estimated in Figure 6 , but the relation is still hypothesis. However, it can be said that FHR below 110 bpm will be parallel to fetal $\mathrm{PaO}_{2}$.

It was requested to estimate the impact of hypoxia, where rapid delivery is indicated but before the loss of LTV. Fetal $\mathrm{PaO}_{2}$ is suitable but its determination is very hard in the labor. Therefore, FHR 
Citation: Maeda K (2014) Electronic and Biologic Simulations of Fetal Heart Rate Changes. J Health Med Informat. 5: e117. doi:10.4172/2157$7420.1000 \mathrm{e} 117$

Page 3 of 3

was used to represent fetal $\mathrm{PaO}_{2}$. The duration of hypoxia was also important factor of the effect of hypoxia. The Hypoxia Index (HI) was created: (The duration of FHR below $110 \mathrm{bpm} /$ the lowest FHR in the bradycardia) $\times 100$. The HI of V-shaped deceleration was $1 / 2$ of U-shaped one.

The HIs of fetal bradycardia in cases of the loss of LTV or brain damage are;

The continuous bradycardia in a placental abruption; $\mathrm{HI}=25$

The brain damage of severe LD with the loss of LTV; HI=25

Intrapartum repeated severe VD followed by the loss of LTV; HI=26

The HI of repeated mild to severe variable decelerations; $\mathrm{HI}=5-10$

From this restricted results, the HI, which indicated C-section before the loss of LTV, will be 20-24. More cases should be studied. A computerized automatic HI determination will be convenient. In addition, non-reactive FHR with preserved LTV diagnosed by ACG, and reduced LTV below the confirmed level by the computer, will be useful to receive C-section before the loss of LTV.

\section{Acknowledgement}

The author is grateful to Mr. TAndo, TOITU, Tokyo, for the efforts on electronic simulations.

\section{References}

1. Maeda K (2013) Actocardiographic analysis of fetal hypoxia detected by the bradycardia, Loss of fetal heart rate acceleration and long term variability. J Health Med Inform.

2. Takahashi $H$ (1990) Studies on the cross correlation of fetal heart rate and fetal movement with actocardiogram. Acta Obstet Gynecol Jpn 42: 443-449.

3. Terao T, Kawashima Y, Noto H, Inamoto Y, Lin TY, et al. (1984) Neurological control of fetal heart rate in 20 cases of anencephalic fetuses. Am J Obstet Gynecol 149: 201-208.

4. Teshima N (1993) Non-reactive pattern diagnosed by ultrasonic Doppler actocardiogram and outcome of the fetuses with non-reactive pattern. Nihon Sanka Fujinka Gakkai Zasshi 45: 423-430.

5. Umezawa J (1976) Studies on the relation between heart rate and $\mathrm{PaO}$ in hypoxic rabbits. A comparative study for fetal heart rate change during labor Acta Obstet Gynecol 28: 1203-1212. 